Supporting Information

\title{
Electronic Band Structure and Ultrafast Carrier Dynamics of Two Dimensional (2D) Semiconductor Nanoplatelets (NPLs) in Presence of Electron Acceptor for Optoelectronic Applications
}

\begin{abstract}
Avisek Dutta $^{\dagger}$, Anusri Medda ${ }^{\dagger}$, Rajesh Bera ${ }^{\dagger}$, Ashima Rawat ${ }^{\ddagger}$, Abir De Sarkar* ${ }^{\ddagger}$ and Amitava Patra*,†,
\end{abstract}

${ }^{\dagger}$ School of Materials Sciences, Indian Association for the Cultivation of Science, Jadavpur, Kolkata-700032, India

Institute of Nano Science and Technology, Habitat Centre, Sector 64, Phase 10, Mohali 160062, India

*To whom correspondence should be addressed. E-mail: msap@iacs.res.in (AP) and abir@inst.ac.in (AD Sarkar)

Phone: (91)-33-2473-4971, Fax: (91)-33-2473-2805 


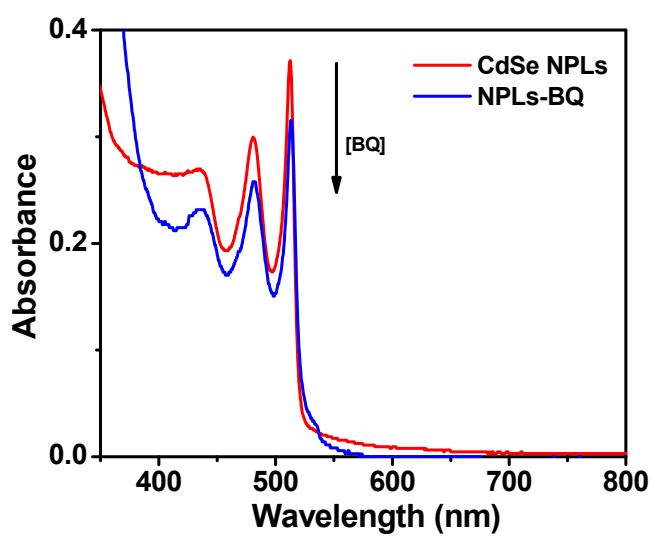

Figure S1.Steady-state absorption spectra of CdSe NPLs with BQ addition (416.25 $\mu \mathrm{M})$.

Table S1. Time-resolved PL decay parameters of the pure CdSe NPLs and corresponding hybrid.

\begin{tabular}{|c|c|c|c|c|}
\hline Systems & $\begin{array}{c}\tau_{1}{ }^{\mathrm{x}}\left(\mathrm{a}_{1}\right)^{\mathrm{y}} \\
(\mathrm{ns})\end{array}$ & $\begin{array}{c}\tau_{2}{ }^{x}\left(a_{2}\right)^{y} \\
(n s)\end{array}$ & $\begin{array}{c}\tau_{3}{ }^{x}\left(a_{3}\right)^{y} \\
(n s)\end{array}$ & $\begin{array}{c}\tau_{\text {average }} \\
\text { (ns) }\end{array}$ \\
\hline CdSe NPLs & $\begin{array}{c}0.36 \\
(0.87)\end{array}$ & $\begin{array}{c}2.8 \\
(0.11)\end{array}$ & $\begin{array}{c}16.2 \\
(0.02)\end{array}$ & $0.95 \pm 0.1$ \\
\hline CdSe NPLs-BQ & $\begin{array}{c}0.2 \\
(0.94)\end{array}$ & $\begin{array}{c}1.45 \\
(0.05)\end{array}$ & $\begin{array}{c}7.92 \\
(0.01)\end{array}$ & $0.34 \pm 0.1$ \\
\hline$x_{ \pm} 4 \%$ and $y_{ \pm} 5 \%$ & & & & \\
\hline
\end{tabular}

\section{Computational details:}

First-principles calculations have been performed within density functional theory (DFT), as implemented in the Vienna Ab Initio simulation package (VASP) ${ }^{1-4}$ code, have been performed. The projected augmented wave $(\mathrm{PAW})^{5}$ pseudo-potentials have been used to describe the ionelectron interactions. The electron exchange and correlation have been treated by the generalized gradient approximation (GGA) as parameterized by Perdew, Burke, and Ernzerhof $(\mathrm{PBE})^{6}$ functional. The electronic wave-functions are expanded using the plane-wave basis set 
with a kinetic energy cut off of $500 \mathrm{eV}$ which ensures the convergence in total energy to a precision of $10^{-6} \mathrm{eV}$ and maximum force on each atom reached less than $0.02 \mathrm{eV} \AA^{-1}$. The Brillouin zone was sampled by $4 \times 4 \times 1 \Gamma$-centered k-point meshes for electronic, dielectric, and optical properties calculations. The absorbance was obtained from the frequency-dependent dielectric function based on the following equation ${ }^{7}$

$$
\alpha_{\mathrm{abs}}=\sqrt{2} \omega\left(\sqrt{\varepsilon_{1}^{2}(\omega)+\varepsilon_{2}^{2}(\omega)}-\varepsilon_{1}(\omega)\right)^{1 / 2}
$$
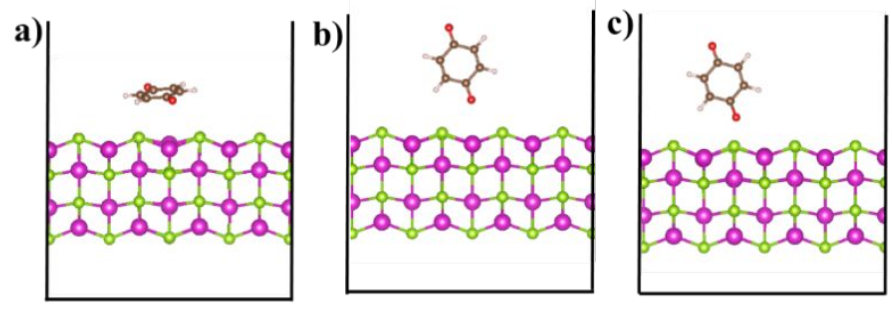

Configuration I

Configuration II

Configuration III

Figure S2. Three different configurations considered for the adsorption of benzoquinone molecule on 4-ML CdSe(110) surface

a)

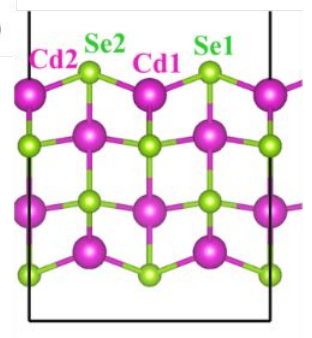

b)

\begin{tabular}{|c|c|}
\hline Atoms & Charges \\
\hline Cd1 & +0.68 \\
\hline Cd2 & +0.68 \\
\hline Se1 & -0.64 \\
\hline Se2 & -0.64 \\
\hline
\end{tabular}

b)

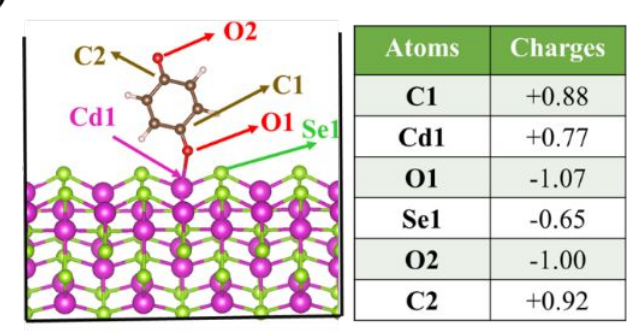

Figure S3. Bader charges on the Carbon(C), Cadmium (Cd), Oxygen (O) and Selenium (Se) atoms in a) $\mathrm{CdSe}(110)$ surface and b) benzoquinone adsorbed on $\mathrm{CdSe}(110)$ surface

Table S2. Calculated excitonic effective mass $\mu_{\mathrm{ex}}\left(\mathrm{m}_{0}\right)$ in units of the rest mass of a free electron $\left(\mathrm{m}_{0}\right)$, macroscopic static dielectric constant $\left(\varepsilon_{\mathrm{r}}\right)$, exciton binding energy $E_{\text {exc }}(\mathrm{eV})$ and Bohr exciton radius $a^{*}(\AA)$ for 4-layer CdSe and benzoquinone adsorbed on 4-layer CdSe. 


\begin{tabular}{|c|c|c|c|c|}
\hline Systems & $\boldsymbol{\mu}_{\mathrm{ex}}\left(\mathbf{m}_{\mathbf{0}}\right)$ & $\boldsymbol{\varepsilon}_{\mathbf{r}}$ & $\boldsymbol{E}_{\text {exc }}(\mathbf{e V})$ & $\boldsymbol{a}^{*}(\mathbf{\AA})$ \\
\hline 4-layer CdSe (110) & 0.13 & 2.80 & 0.96 & 2.76 \\
\hline $\begin{array}{c}\text { Benzoquinone/4-layer } \\
\text { CdSe(110) }\end{array}$ & 0.13 & 2.98 & 0.79 & 3.05 \\
\hline
\end{tabular}

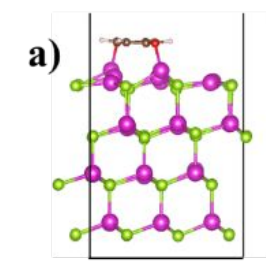

b)
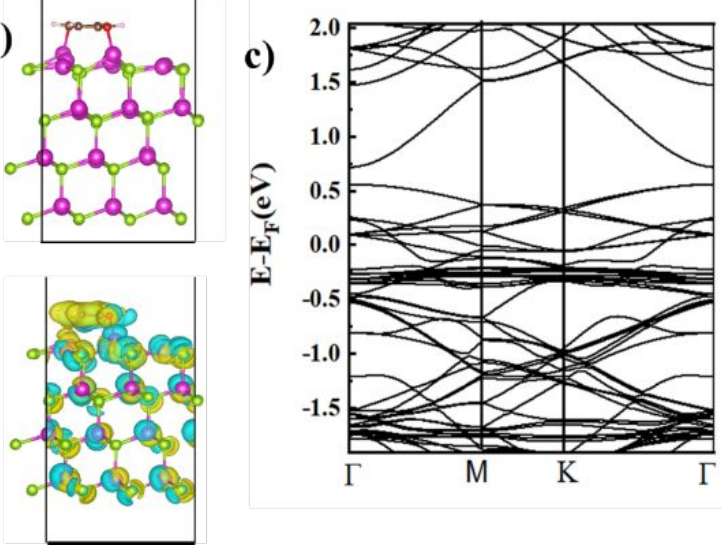

Figure S4. a) Benzoquinone molecule adsorbed on 4-ML CdSe(111) surface, b) 3D iso-surface of charge density difference profile: yellow (cyan) color indicates spatial regions of charge accumulation (depletion) at an iso-surface value of $0.01 \mathrm{e} / \AA^{3} \mathrm{c}$ ) Bandstructure of 4-ML CdSe(111).

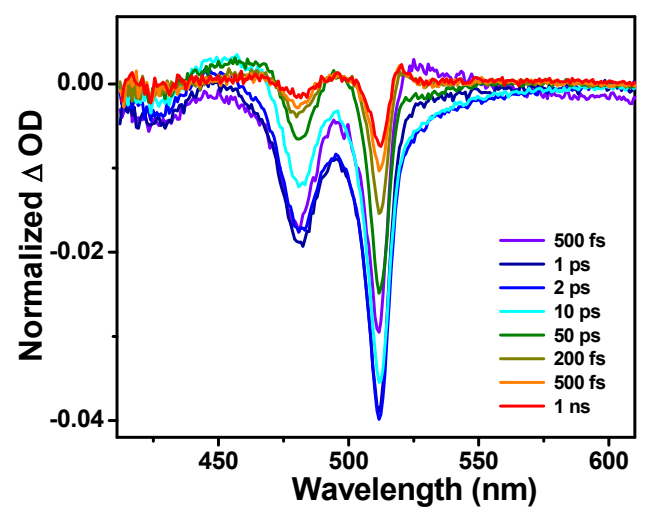

Figure S5. Transient absorption (TA) spectra of pure CdSe NPLs with delay time from 500 fs to $1 \mathrm{~ns}$. 


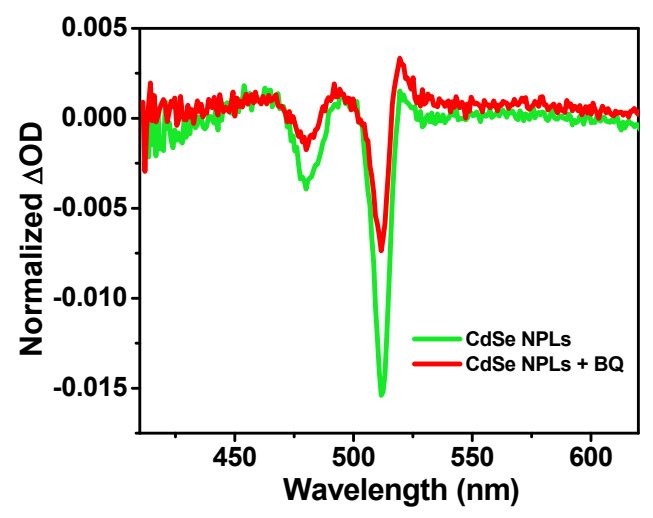

Figure S6. The TA spectrum of pure CdSe NPLs (green) and CdSe NPLs with $416 \mu \mathrm{M}$ of BQ (red) at $200 \mathrm{ps}$ after excitation at $400 \mathrm{~nm}$.

Table S3. Fitting parameters for pure CdSe NPLs and CdSe NPLs-BQ hybrid

\begin{tabular}{lllll}
\hline Systems & $\begin{array}{l}\tau^{\mathbf{g}} \\
(\mathbf{p s})\end{array}$ & $\begin{array}{l}\tau_{\mathbf{r}}(\mathbf{a} \mathbf{1} \%) \\
(\mathbf{p s})\end{array}$ & $\begin{array}{l}\tau_{\mathbf{r}}\left(\mathbf{a}_{\mathbf{2}} \%\right) \\
(\mathbf{p s})\end{array}$ & $\tau_{\mathbf{r}}\left(\mathbf{a}_{\mathbf{3}} \%\right)$ \\
\hline CdSe NPLs & 0.4 & - & $\begin{array}{l}47.0 \pm 2.3 \\
(55.5 \%)\end{array}$ \\
CdSe-BQ & 0.3 & $2.1 \pm 0.1$ & $46.1 \pm 2.3$ & $>1 \mathrm{~ns}$ \\
& & $(6.2 \%)$ & $(65.9 \%)$ & $>1 \mathrm{~ns}$ \\
& & & $(27.9 \%)$ \\
\hline
\end{tabular}

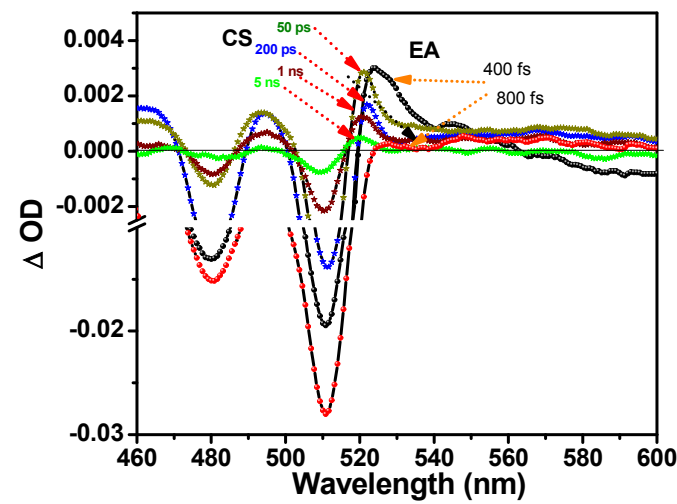

Figure S7. Transient absorption (TA) spectra evolution of the hybrid from 0.4 ps to 5 ns showing EA and CS features. 


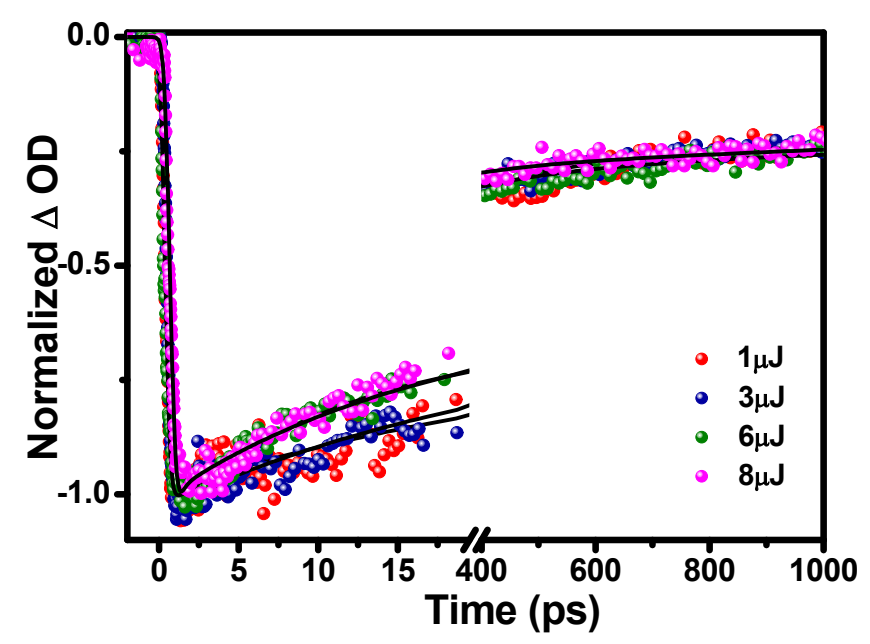

Figure S8. Pump power dependence of the transient bleach decay kinetics in the NPLs-BQ hybrid.
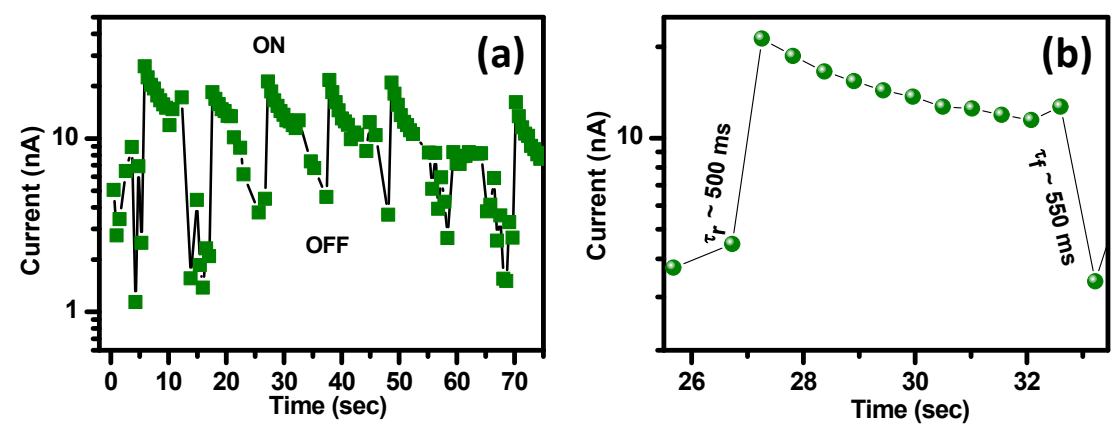

Figure S9. (a) Optical switching characteristics of pure NPLs based device recorded by switching the light source at $2 \mathrm{~V}$ bias. (d) Rise and fall time of a single on-off cycle of pure sample.

\section{REFERENCES}

1. Kresse, G.; Furthmüller, J. Efficient Iterative Schemes for ab initio Total-Energy Calculations using a Plane-Wave Basis Set. Phys. Rev. B 1996, 54, 11169-11186.

2. Kresse, G.; Hafner, J. Ab initio Molecular-Dynamics Simulation of the Liquid-Metal-Amorphous-Semiconductor Transition in Germanium. Phys. Rev. B 1994, 49, 14251-14269.

3. Kresse, G.; Hafner, J. Ab initio Molecular Dynamics for Liquid Metals. Phys. Rev. B 1993, $47,558-561$.

4. Kresse, G.; Furthmüller, J. Efficiency of ab-initio Total Energy Calculations for Metals and Semiconductors using a Plane-Wave Basis Set. Comput. Mater. Sci. 1996, 6, 15-50.

5. Blöchl, P. E. Projector Augmented-Wave Method. Phys. Rev. B 1994, 50, 17953-17979. 
6. Perdew, J. P.; Burke, K.; Ernzerhof, M. Generalized Gradient Approximation Made Simple. Phys. Rev. Lett. 1996, 77, 3865-3868.

7. Gajdoš, M.; Hummer, K.; Kresse, G.; Furthmüller, J.; Bechstedt, F. Linear optical Properties in the Projector-Augmented Wave Methodology. Phys. Rev. B 2006, 73, 045112. 